\title{
A Three-Level MOSFET Inverter for Low-Power Drives
}

\author{
Brian A. Welchko, Member, IEEE, Maurício Beltrão de Rossiter Corrêa, Member, IEEE, and \\ Thomas A. Lipo, Life Fellow, IEEE
}

\begin{abstract}
This paper proposes operating a three-level neutralpoint-clamped (NPC) inverter using a two-level pulsewidth-modulation method. This allows for the clamping diodes to be rated at a fraction of the main switches due to their low average current requirement. The use of a bootstrap charge pump as a low-cost method to obtain the isolated gate drive power supplies is extended for use with the NPC topology. Using this control method and circuits, an inverter based on high-volume, low-cost, low-voltage power MOSFETs is experimentally demonstrated as a possible economic alternative to an insulated-gate-bipolar-transistor-based drive for 120-Vrms-supplied systems.
\end{abstract}

Index Terms-Adjustable-speed drives, bootstrap, charge pump, inverters, multilevel systems, pulsewidth modulation.

\section{INTRODUCTION}

$\mathbf{T}$ HE three-level neutral-point-clamped (NPC) inverter [1] shown in Fig. 1 has evolved into the standard for medium-voltage motor drive systems as evidenced by the commercial availability of medium voltage drives based on both integrated gate commutated transistor (IGCT) [2] and high-voltage insulated gate bipolar transistor (IGBT) devices [3]. The topology has two important attributes that make it well suited to this market: lower harmonic content than a standard two-level inverter, and the fact that the main switching devices are required to block only one-half of the dc-bus voltage. This latter attribute has traditionally been exploited to allow for higher voltage drives since device voltages have been limited. However, it also implies that a drive of a given voltage can be obtained with lower voltage devices by employing the NPC topology.

Low-voltage power MOSFETs have achieved a significant (approximately $4-5: 1$ ) cost advantage in terms of dollars per ampere over IGBTs due to their very widespread use in the automotive and power supply industries. This paper investigates the feasibility of a low-power motor drive employing 150-V MOSFETs and the NPC topology. Since the NPC topology requires

Manuscript received August 22, 2002; revised October 14, 2003. Abstract published on the Internet January 14, 2004. This paper was presented at the 28th Annual Conference of the IEEE Industrial Electronics Society, Seville, Spain, November 5-8, 2002.

B. A. Welchko was with the Department of Electrical and Computer Engineering, University of Wisconsin, Madison, WI 53706 USA. He is now with General Motors Advanced Technology Center, Torrance, CA 90505 USA (e-mail: bwelchko@ieee.org)

M. B. de Rossiter Corrêa is with the Departamento de Engenharia Elétrica, Universidade Federal da Paraíba, 58109-970 Campina Grande, Brazil (e-mail mbeltrao@dee.ufcg.edu.br).

T. A. Lipo is with the Department of Electrical and Computer Engineering, University of Wisconsin, Madison, WI 53706 USA (e-mail: lipo@engr wisc.edu)

Digital Object Identifier 10.1109/TIE.2004.825337 twice as many switches, the cost savings afforded by using inexpensive MOSFETs as the main switching devices can be eliminated by the extra gate drivers and clamping diodes that the NPC topology requires. In an effort to overcome this problem, this paper proposes a gate driver circuit designed around inexpensive discrete components. It also proposes a four-level bootstrap charge pump scheme, which eliminates the need for isolated gate drive power supplies. It further proposes a new control method for the NPC inverter that allows for the clamping diodes to be rated at only a fraction of the current rating of the main switches. This combination results in a low-power drive that may be competitive in terms of component cost when compared to an IGBT-based drive. The concept has important commercial implications since cost has been the largest drawback to the widespread consumer adoption of low-voltage drives [4].

\section{OPERATING PRINCIPLE}

The NPC inverter is capable of connecting the load to the upper bus (level 2, $S_{x 1}, S_{x 2}$ on), the dc-bus neutral point (level 1, $S_{x 2}, S_{x 3}$ on), and the lower bus rail (level 0, $S_{x 3}$, $S_{x 4}$ on) [5], where the subscript $x$ represents either phase $a$, $b$, or $c$, as shown in Fig. 1. When outputting a level 0, one of the clamping diodes $\left(D_{x 1}, D_{x 2}\right)$ is conducting the phase current depending on the current polarity. If the converter is actively controlled to quickly transition through the level-1 output state, which is a requirement for proper commutation, the clamping diodes will only carry a small average current. As a result, they can be rated at a fraction of the current rating of the main switches, reducing their cost significantly. The use of high instantaneous currents but low average currents in low-current-rated devices has been previously applied to soft-switching topologies as a cost-saving measure [6]. Hence, the inverter is actively operated as a two-level inverter using the space-vector diagram shown in Fig. 2. Operation by actively using the level-1 switching states shown in Fig. 2 is, therefore, not allowed for this NPC inverter with smaller diode ratings.

Since many microcontrollers and motor control digital signal processors (DSPs) have embedded hardware or software to execute the traditional two-level pulsewidth-modulation (PWM) method, it is desirable to formulate the proposed PWM method for the NPC inverter to take advantage of these embedded functions. This would eliminate the need to encode a three-level PWM modulator. This embedded two-level PWM architecture in the controller is well suited to the proposed PWM method since the dead time that is inserted by the embedded architecture can be used to generate the level-1 output for this NPC inverter. The high- and low-level outputs of the two-level modu- 


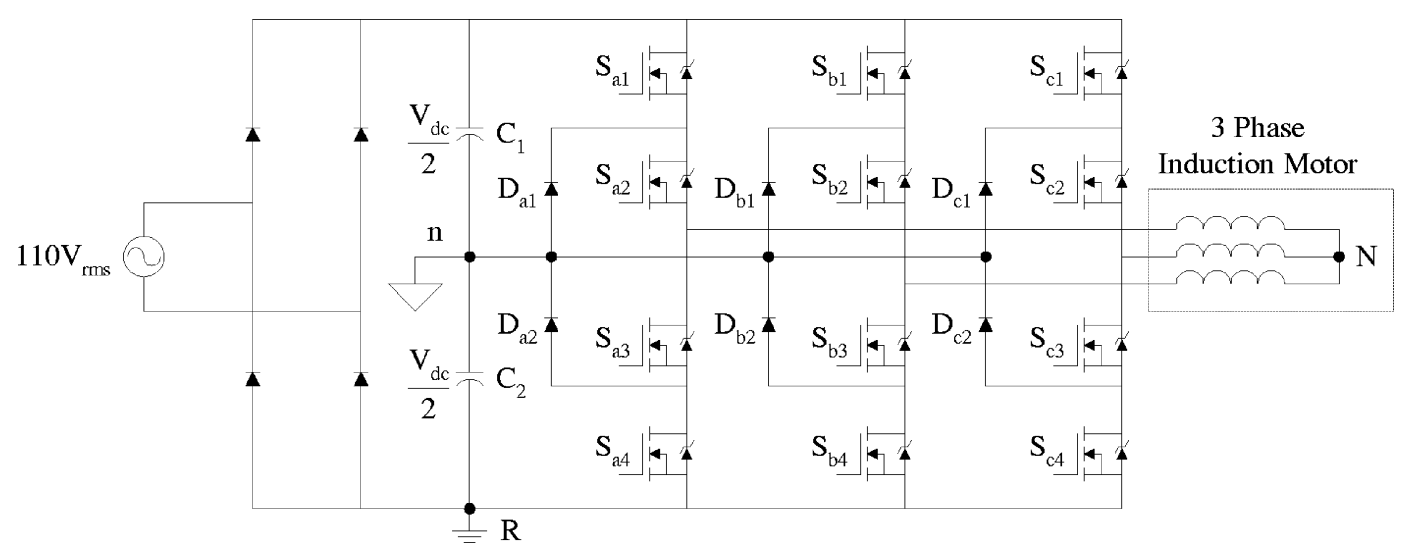

Fig. 1. Neutral-point-clamped three-level inverter driving a three-phase induction motor.

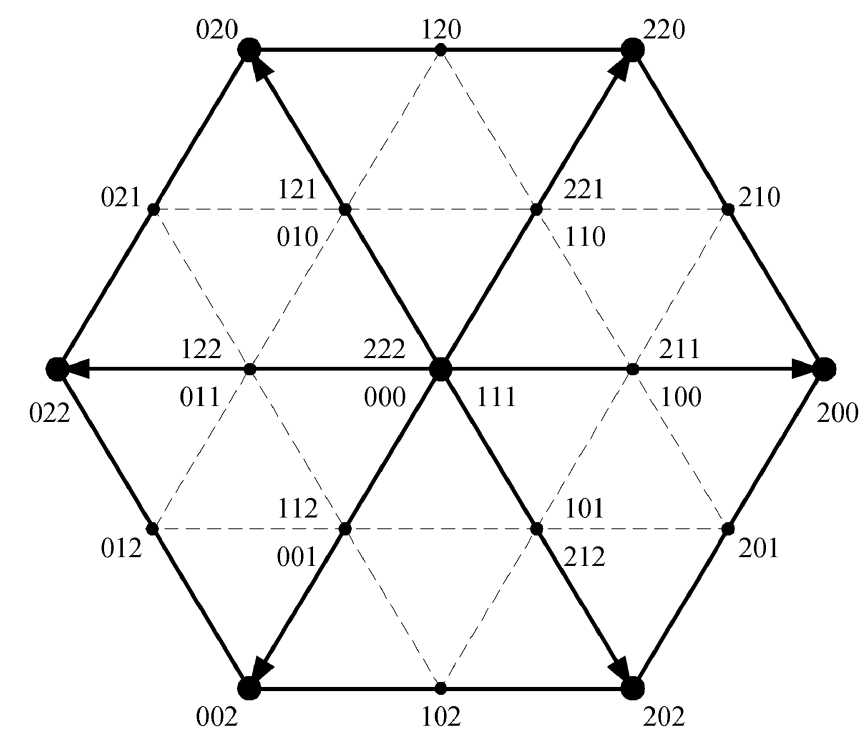

Fig. 2. Space-vector diagram with two-level operation in bold solid lines; traditional three-level diagram shown dashed.

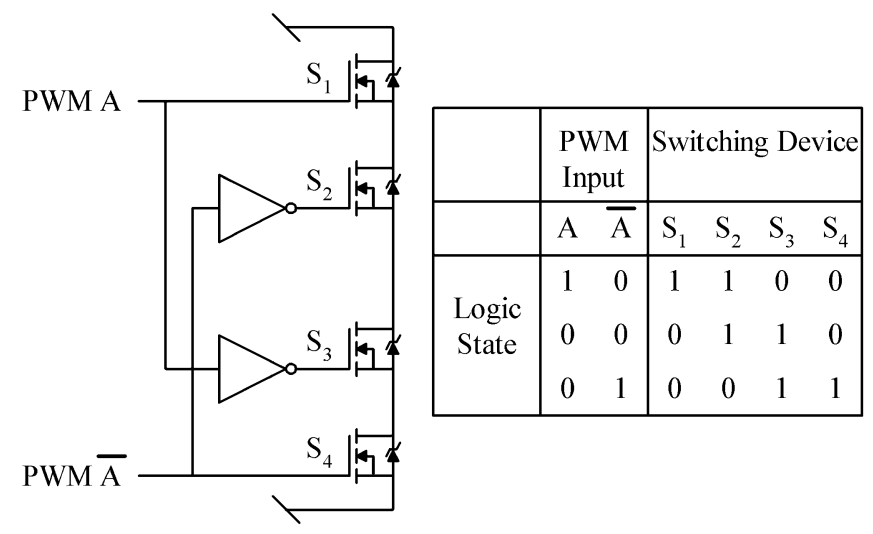

Fig. 3. Generation of the four gate signals using the outputs of a two-level PWM modulator.

lator are used as level-2 and level-0 outputs for the proposed PWM method, respectively. Fig. 3 shows how the two-level modulator can be used in conjunction with two low-cost inverter gates to generate the four required gate signals for each phase of the NPC inverter.

It is important to note that employing logic gates to derive four PWM gate signals from the two signals from the modulator implies that there will be no inherent software-based dead time in this three-level NPC inverter. The consequences of this can be minimized with proper design of the converter. For the proposed converter, careful attention was paid to the gate driver design so that turn-on and turn-off switching and delay times were matched so that a shoot-through condition is avoided. As an alternative, or as an extra precaution, a passive dead-time lockout circuit using discrete components could be employed.

\section{GATE DRIVE CIRCUITRY}

The gate drive design for the proposed control method for the NPC inverter requires careful consideration of economic issues for the target low cost application and timing issues relating to dead-time and shoot-through issues as discussed in the following two sections.

\section{A. Gate Drive Power Supplies}

Charge pump or bootstrap circuits have been the circuits of choice to achieve the gate drive power supplies for low-cost twolevel inverters since they provide the required power supplies referenced to the ground of each switch without the use of expensive transformers to create each supply. This paper extends their use to the three-level NPC inverter. This four-level bootstrap charge pump shown in Fig. 4 works as follows. A single low-voltage gate drive power supply, $V_{G 4}$, is referenced to the negative dc bus. When the switch $S_{4}$ is turned on, $C_{G 3}$ is charged through diode $D_{G 3}$. Since $S_{3}$ now has a power supply, $S_{3}$ and $S_{4}$ can both be turned on. This will charge $C_{G 2}$ through $D_{G 2}$ and recharge $C_{G 3}$. Finally, $C_{G 1}$ is charged by $C_{G 2}$ through $D_{G 1}$ by turning on $S_{2}$. As a result, after this startup sequence, each of the gate drive power supplies is charged during each PWM cycle with the size of the capacitor determining the length of holdup time that each switch can remain on without the respective gate drive supply recharging. Due to the holdup time requirements, it is unlikely that this bootstrap charge pump circuit would be practical to be compatible with six-step operation of the drive. However, this limitation is common to all bootstrap circuits such as this.

When using the proposed control method, $C_{G 3}$ and $C_{G 2}$ are charged during a level-0 output, $C_{G 2}$ and $C_{G 1}$ are charged during a level-1 output, and $C_{G 1}$ is charged during a level-2 output.

This bootstrap charge pump has several practical restrictions that must be considered during the design process. The charging 


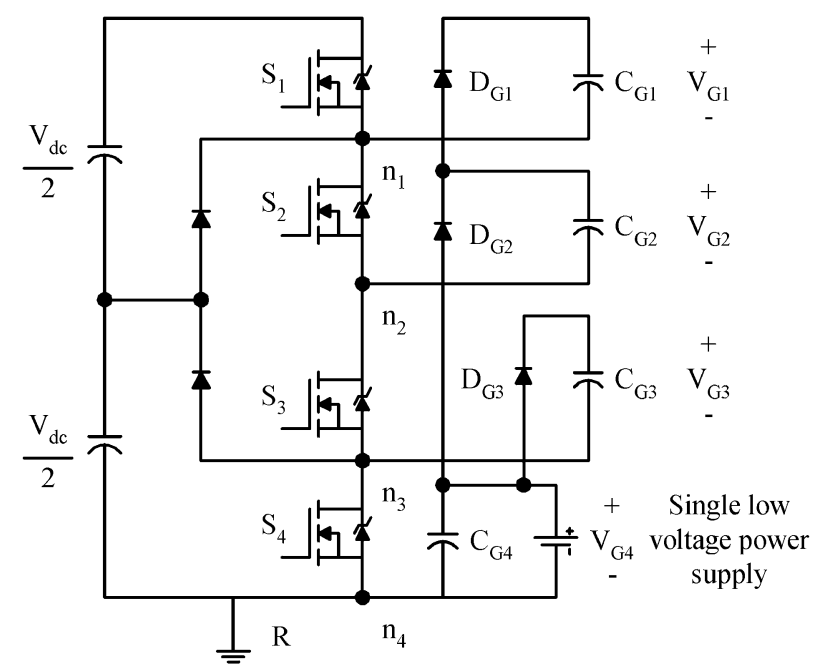

Fig. 4. Phase leg shown with connections of the four-level bootstrap charge pump to obtain the gate drive power supplies.

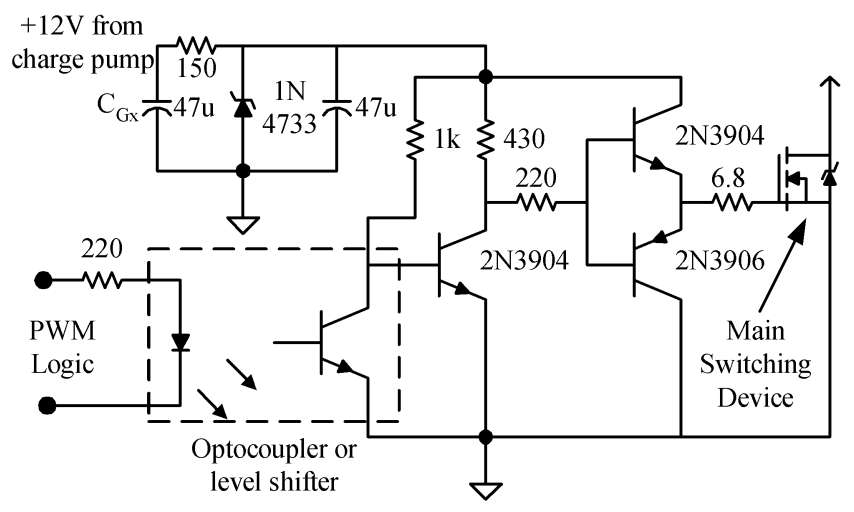

Fig. 5. Individual gate driver circuit design.

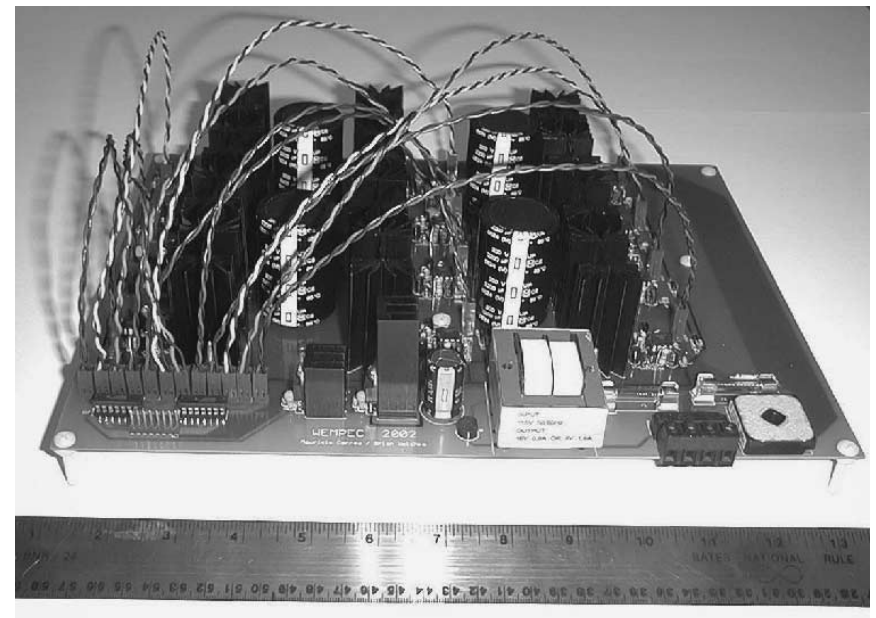

Fig. 6. The constructed three-level NPC inverter.

diodes need to be rated to block the full dc-link voltage instead of only half like the main switching devices. Economically, this is of no consequence as the price of low-power switching diodes is not dependant on voltage. It is also important that the worst case device voltage drops be considered when deciding what voltage to use for the one independent supply of $V_{G 4}$. For example, the

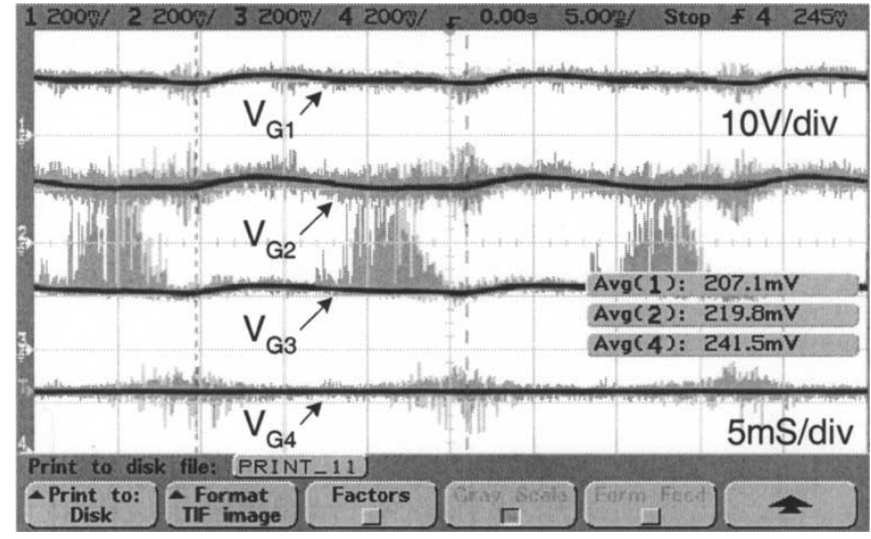

Fig. 7. Preregulated gate drive supply voltages obtained via the bootstrap charge pump. Ch1 $-\mathrm{V}_{\mathrm{G} 1}, \mathrm{Ch} 2-\mathrm{V}_{\mathrm{G} 2}, \mathrm{Ch} 3-\mathrm{V}_{\mathrm{G} 3}, \mathrm{Ch} 4-\mathrm{V}_{\mathrm{G} 4} .10 \mathrm{~V} / \mathrm{div}$.

voltage ultimately at $C_{G 1}$ will be the supply voltage $V_{G 4}$ minus three main switching device drops $\left(S_{4}, S_{3}\right.$, and $\left.S_{2}\right)$ and two charging diode drops $\left(D_{G 2}\right.$ and $\left.D_{G 1}\right)$. Furthermore, the minimum capacitance chosen for $C_{G 3}$ and $C_{G 2}$ must, for practical purposes, be larger than the other capacitors since they serve as the source of charge during parts of the PWM control cycle.

\section{B. Gate Driver Topology}

A gate drive circuit based on low-cost discrete components was designed as shown in Fig. 5. The PWM input signal to the gate driver was obtained through an optocoupler for purposes of this paper. While this provides superior performance, it is likely that some other form of level shifter or pulse transformer would provide the required performance at a lower cost. The gate driver itself is composed of three small TO-92 n-p-n and $\mathrm{p}-\mathrm{n}$-p transistors and four resistors.

For this paper, the gate drive power supply voltage was set to a nominal $12 \mathrm{~V}$ referenced to the bus ground $R$ at the lower most switch. In order to obtain consistent switching performance for the four gate drivers and switches in each phase, the nominal $12-\mathrm{V}$ gate drive power supply obtained by the bootstrap charge pump was regulated via a low-cost 5-V Zener diode in series with a resistor. This regulated voltage helps ensure consistent turn-on and turn-off delay times between the devices as the miller plateau voltage of the MOSFETs used was approximately $3 \mathrm{~V}$, hence, the gate drive current during charging and discharging of this gate capacitance is not substantially different.

\section{EXPERIMENTAL RESULTS}

To verify the operation of the proposed converter and control method, a hardware prototype was constructed with $150-\mathrm{V} 12-\mathrm{A}$ IRL3215 MOSFETs and 200-V 1-A UF1003 clamping diodes. The prototype inverter is shown in Fig. 6. Experimental results of the three-phase system are shown in Figs. 7-14. All of the results presented here were obtained with the inverter connected to the induction motor described in the Appendix. The inverter was operated using an open-loop volts/hertz control method. The motor was unloaded, operating at $60 \mathrm{~Hz}$ and at rated flux. The average dc-bus voltage was $152 \mathrm{~V}$ obtained from a nominal $120-\mathrm{V}_{\text {rms }} 60-\mathrm{Hz}$ input. 


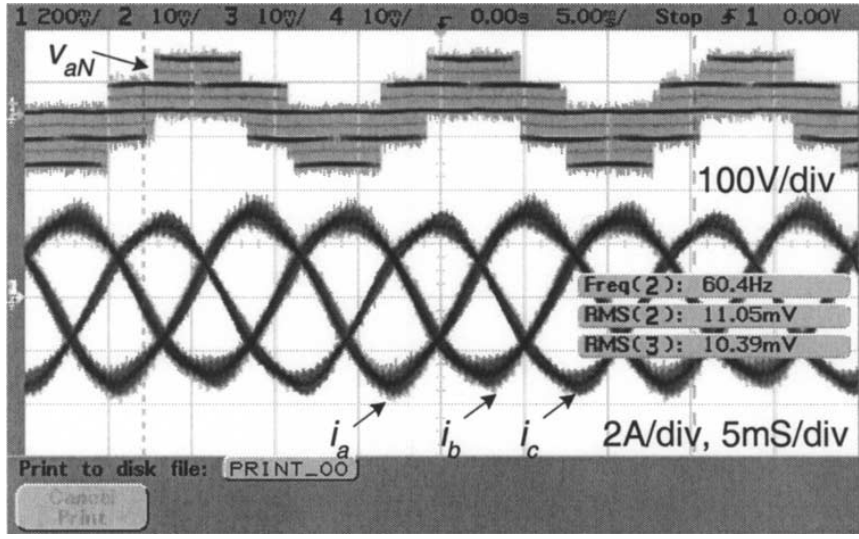

Fig. 8. Motor phase- $a$ voltage and phase currents. Ch1 $-v_{a N}(100 \mathrm{~V} / \mathrm{div})$, $\mathrm{Ch} 2-i_{a}, \mathrm{Ch} 3-i_{b}, \mathrm{Ch} 4-i_{c}(2 \mathrm{~A} / \mathrm{div})$.

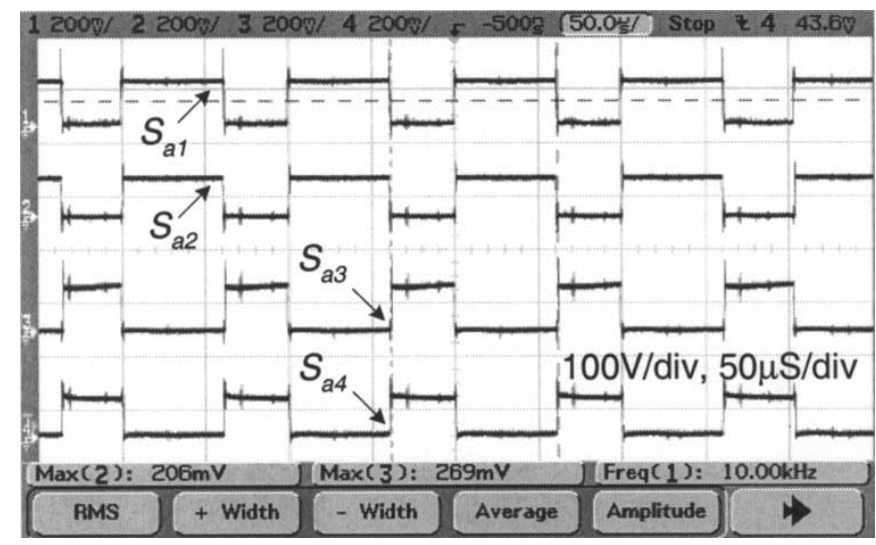

Fig. 9. Drain-to-source voltages during a typical PWM cycle. Ch1- $\mathrm{S}_{1}$, $\mathrm{Ch} 2-\mathrm{S}_{2}, \mathrm{Ch} 3-\mathrm{S}_{3}, \mathrm{Ch} 4-\mathrm{S}_{4} .100 \mathrm{~V} / \mathrm{div}$.

Fig. 7 shows the preregulated output voltages of the bootstrap charge pump circuit. This result verifies the proposed bootstrap charge pump circuit as a low-cost method to obtain the individually referenced gate drive power supplies. In the figure, some variation with the output fundamental output frequency $(60 \mathrm{~Hz})$ is seen due to the change in load current, and hence, switch voltage drops throughout the cycle. On average, the voltage drops from $12.07 \mathrm{~V}$ at the lower referenced supply to $10.35 \mathrm{~V}$ at the upper most referenced supply due to these device drops. The noise seen in this figure is a consequence of using the main switches in the charge pump circuit since any ringing on their drain-to-source voltage due to switching is propagated through to the $12-\mathrm{V}$ outputs of the charge pump.

Fig. 8 shows the phase voltage and current waveforms produced by the inverter. The fundamental phase output voltage had a value of $45.4 \mathrm{~V}_{\mathrm{rms}}$ as measured by a spectrum analyzer. The phase currents shown are high quality sinusoids and are well balanced despite the open-loop nature of the control algorithm.

Fig. 9 shows the drain-to-source voltages of the phase $a$ devices during a typical PWM cycle. Some coupling on the dc-bus voltage is seen from the operation of the other phases as evidenced by the voltage spikes when this phase is not switching. The switching frequency of the converter was $10 \mathrm{kHz}$. From this picture, it is not possible to discern the presence of the level 1 output state of the converter during the transition from a level-0 to level-2 or level-2 to level-0 output.

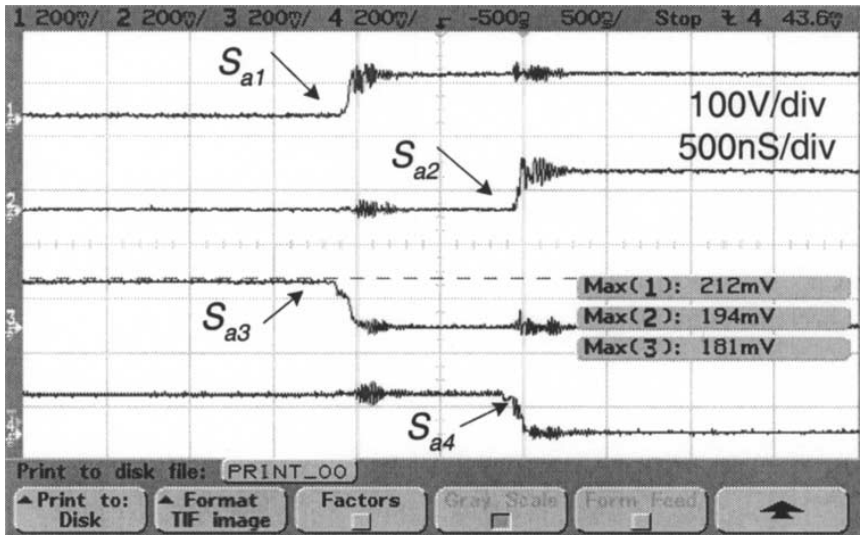

Fig. 10. Drain-to-source voltages during a level 2 to level 0 output transition. Ch1 $-\mathrm{S}_{1}, \mathrm{Ch} 2-\mathrm{S}_{2}, \mathrm{Ch} 3-\mathrm{S}_{3}, \mathrm{Ch} 4-\mathrm{S}_{4} .100 \mathrm{~V} / \mathrm{div}$.

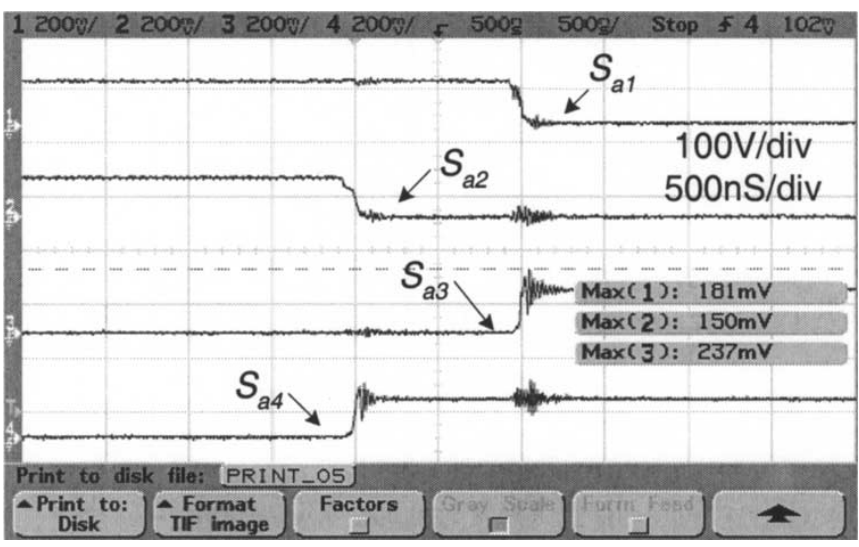

Fig. 11. Drain-to-source voltages during a level 0 to level 2 output transition. $\mathrm{Ch} 1-\mathrm{S}_{1}, \mathrm{Ch} 2-\mathrm{S}_{2}, \mathrm{Ch} 3-\mathrm{S}_{3}, \mathrm{Ch} 4-\mathrm{S}_{4} .100 \mathrm{~V} / \mathrm{div}$.

Figs. 10 and 11 show details of the switching behavior of the converter with Fig. 10 showing the transitions from a level-2 to level- 0 output and Fig. 11 showing the transition from a level-0 to level-2 output. As seen in the figures, the time duration for the level-1 output state was set to $1 \mu \mathrm{s}$. This represents a loss of $2 \%$ of the output voltage capacity of the inverter since there are two transitions per PWM cycle. Due to the clamping diodes, the overshoot of the drain-to-source voltage at turn-off is limited by the bus capacitor voltages. In that sense, the bus capacitors provide a built-in snubber for the switches. It is important to note, that because of the stray inductance between this snubber and the devices, along with the poor high frequency properties of electrolytic capacitors, a local snubber circuit may be needed for a particular layout and choice of devices. For the constructed prototype, no such local snubber circuit was used. Due to the clamping diodes, switching transients on one switch will affect the voltage seen by the adjacent series switch. The devices in the figures are switching simultaneously and no evidence of shootthrough is present.

Fig. 12 shows the bus capacitor voltages over a typical fundamental output cycle. As seen in the figure, there is an imbalance between the two capacitor voltages of $10 \%$. In the circuit configuration, the capacitor neutral voltage is not referenced to a fixed quantity. Without using the level-1 output as an active state, the voltage unbalance cannot be actively removed. It should also be pointed out that, by not using the active 1 state, the voltage bal- 


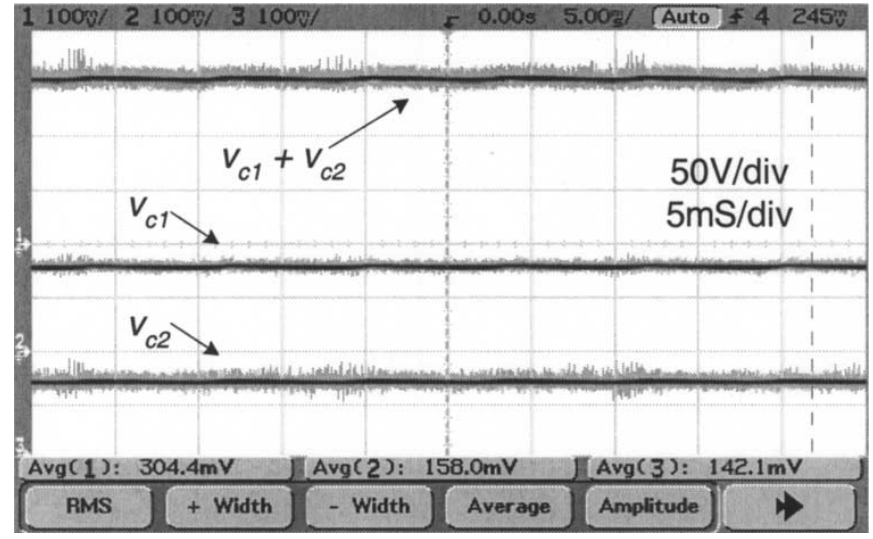

Fig. 12. Bus capacitor voltages. Ch1-v $v_{c 1}+v_{c 2}$, Ch2- $v_{c 1}$, Ch3-v $v_{c 2}$ $50 \mathrm{~V} /$ div.

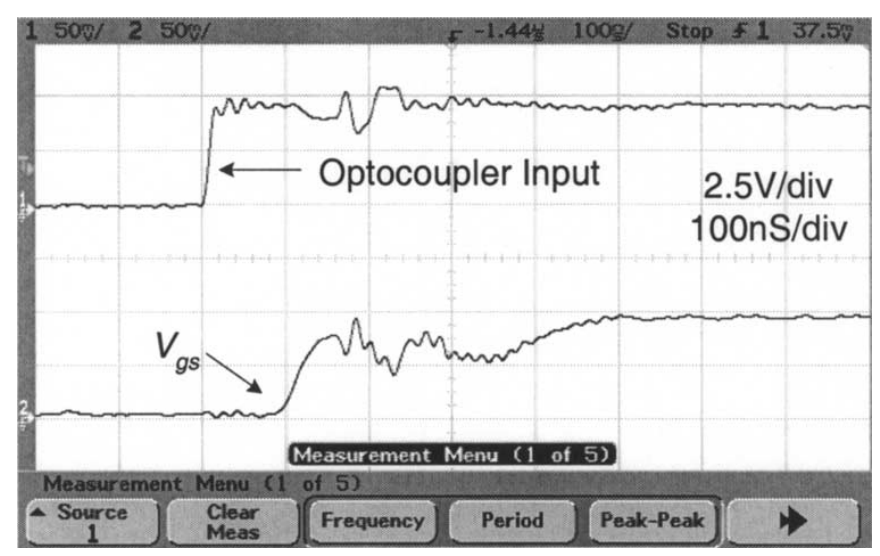

Fig. 13. Gate drive signals during turn-on. Ch1-optocoupler input, $\mathrm{Ch} 2-v_{g s}$. $2.5 \mathrm{~V} /$ div.

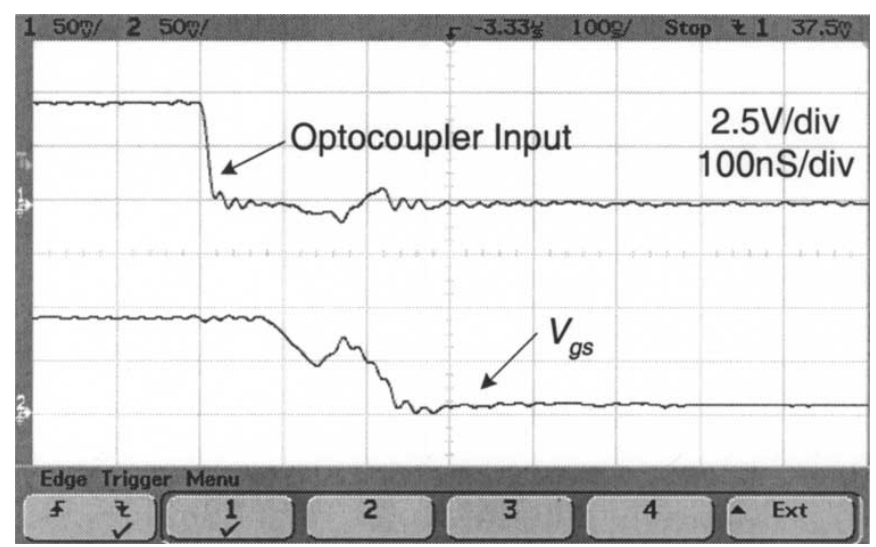

Fig. 14. Gate drive signals during turn-off. Ch1—optocoupler input, Ch2- $v_{g s} .2 .5 \mathrm{~V} / \mathrm{div}$.

ancing issue is the same as it would be for any two-level converter employing series capacitors in the dc link. For the constructed circuit, small $10-\mathrm{k} \Omega$ resistors were used as bleeder resistors on each capacitor. These resistors provided the only balancing necessary for the constructed drive.

Figs. 13 and 14 show the switching performance of the gate drive circuit from the input to the optocoupler to the gate-tosource voltage of one of the main switches. From the figures, it takes about $500 \mathrm{~ns}$ to switch on and $300 \mathrm{~ns}$ to switch off. The difference is due to the plateau voltage of the device which is
$3 \mathrm{~V}$. During switch-off, the voltage across the gate resistor is $2 \mathrm{~V}$ while it is $3 \mathrm{~V}$ during turn-on. This difference was used as an effective dead-time control since it is desirable for the devices to turn off faster than they turn on in order to avoid any possibilities for shoot-through failures.

\section{CONCLUSION}

This paper has proposed a new PWM control method for a three-level NPC inverter. It operates the three-level inverter effectively as a two-level inverter. This allows for a significant reduction in the rating requirements of the clamping diodes, which would result in a lower cost implementation of this topology. The paper also extends the NPC topology to the use of a bootstrap charge pump circuit as a method to obtain the required independently referenced gate drive power supplies. This bootstrap charge pump circuit eliminates the need for individual power transformers for each of the gate drive supplies which significantly reduces the cost and size of these required supplies. With these proposed methods, a low-power motor drive was constructed using inexpensive high-volume low-voltage power MOSFETs and other low-cost discrete components. Thus, the paper demonstrates the possibility of basing a low-power motor drive around inexpensive power MOSFET switches that previously could not be used due to voltage limitations.

While the proposed converter was constructed using discrete power devices, it should be noted that the two-level control principle and four-level bootstrap charge pump make this topology attractive for integration into a single device package much like a standard six-pack arrangement. With the lower voltage rating requirement of the main switches, and the familiar and standard two-level control principles, this topology could become an economical alternative to a standard two-level inverter depending on what future power switching devices are developed and manufactured. This is important to note since the trend of integration into standard packages and automated manufacturing focus attention on performance and total cost while making the actual topology inside the package of lesser importance.

\section{APPENDIX \\ MACHINE PARAMETERS}

The 1-hp 5000-r/min two-pole 115/230-V three-phase squirrel-cage-rotor dual-wound-stator induction machine used for this paper had the following characteristics when configured for low-voltage operation:

$$
\begin{aligned}
R_{1} & \approx 0.50 \Omega \\
R_{2} & \approx 0.35 \Omega \\
L_{1} & \approx L_{2} \approx 1.9 \mathrm{mH} \\
L_{m} & \approx 55 \mathrm{mH}
\end{aligned}
$$

\section{ACKNOWLEDGMENT}

The authors would like to acknowledge the motivation provided by the member companies of the Wisconsin Electric Machines and Power Electronics Consortium (WEMPEC) at the University of Wisconsin, Madison, and their commitment to support the research activities of CPES. The authors would also like to thank the Motorola Corporation for providing the DSP 
56F805 EVM board and CodeWarrior 4.0 software development kit used to control the inverter for this paper.

This work made use of shared facilities supported by the Center for Power Electronics Systems (CPES). CPES is a National Science Center Engineering Research Center under Award EEC-9731677.

\section{REFERENCES}

[1] A. Nabae, I. Takahashi, and H. Akagi, "A new neutral-point clamped PWM inverter," IEEE Trans. Ind. Applicat., vol. IA-17, pp. 518-523, Sept./Oct. 1981.

[2] P. K. Steimer, J. K. Steinke, H. E. Grüning, and S. Conner, "A reliable, interface-friendly medium voltage drive based on the robust IGCT and DTC technologies," in Conf. Rec. 34th IEEE-IAS Annu. Meeting, vol. 3, Phoenix, AZ, 1999, pp. 1505-1512.

[3] R. Sommer, A. Mertens, M. Griggs, H.-J. Conraths, M. Bruckmann, and T. Greif, "New medium voltage drive systems using three-level neutral point clamped inverter with high voltage IGBT," in Conf. Rec. 34th IEEE-IAS Annu. Meeting, vol. 3, Phoenix, AZ, 1999, pp. 1513-1519.

[4] K. Phillips, "Power electronics: Will our current technical vision take us to the next level of AC drive product performance?," in Conf. Rec. 35th IEEE-IAS Anпи. Meeting, vol. 1, Rome, Italy, 2000, pp. P1-P9.

[5] B. P. McGrath, D. G. Holmes, and T. A. Lipo, "Optimized space vector switching sequences for multilevel converters," IEEE Trans. Power Electron., vol. 18, pp. 1293-1301, Nov. 2003.

[6] Y. Li, F. C. Lee, J. Lai, and D. Boroyevich, "A low-cost three-phase zero-current-transition inverter with three auxiliary switches," in Proc. IEEE PESC'00, vol. 1, Galway, Ireland, 2000, pp. 527-563.

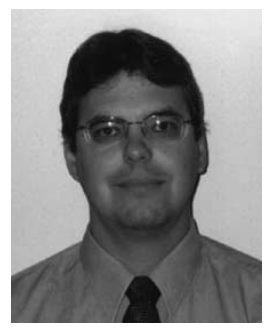

Brian A. Welchko (S'98-M'04) received the B.S. and M.S. degrees from Ohio University, Athens, in 1994 and 1996,respectively, and the Ph.D. degree from the University of Wisconsin, Madison, in 2003, all in electrical engineering.

During his Ph.D. studies, he worked summers with the Otis Elevator Company, Farmington, CT, in 1997 and 1998, and with General Motors Advanced Technology Center (GMATC), Torrance, CA, in 2000, 2001, and 2002. He joined GMATC in 2004. His current research interests are in novel power converter topologies and control methods applied to interior permanent-magnet synchronous machines.

Dr. Welchko regularly reviews IEEE conference and TRANSACTIONS papers in his area of expertise and served as the Technical Program Co-Chair for the 2003 IEEE International Electric Machines and Drives Conference (IEMDC), which was held in Madison, WI.

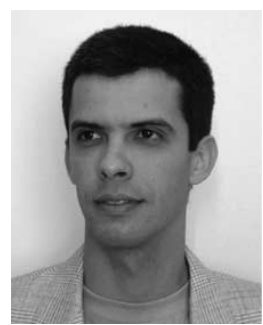

Maurício Beltrão de Rossiter Corrêa (S'97-M'03) was born in Maceió, Brazil, in 1973. He received the B.S., M.S., and Ph.D. degrees in electrical engineering from the Federal University of Paraíba, Campina Grande, Brazil, in 1996, 1997, and 2002, respectively.

Since 1997, he has been a faculty member of the Coordenação de Ensino Tecnológico-Centro Federal de Educação Tecnológica de Alagoas, Palmeira dos Indios, Brazil. His research interests include power electronics and electrical drives.

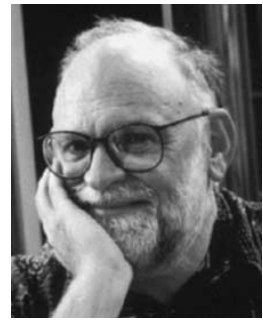

Thomas A. Lipo (M'64-SM'71-F'87-LF'04) was born in Milwaukee, WI. He received the B.E.E. and M.S.E.E. degrees from Marquette University, Milwaukee, WI, in 1962 and 1964, respectively, and the $\mathrm{Ph} . \mathrm{D}$. degree in electrical engineering from the University of Wisconsin, Madison, in 1968.

From 1969 to 1979, he was an Electrical Engineer in the Power Electronics Laboratory of Cooperate Research and Development of the General Electric Company, Schenectady, NY. He became a Professor of Electrical Engineering at Purdue University, West Lafayette, IN, in 1979, and in 1981, he joined the University of Wisconsin, Madison, in the same capacity, where he is presently the W. W. Grainger Professor for Power Electronics and Electrical Machines, Co-Director of the Wisconsin Electric Machines and Power Electronics Consortium, and Director of the Wisconsin Power Electronics Research Center. He has authored 400+ published technical papers and is the holder of 32 U.S. patents.

Dr. Lipo has received the 1986 Outstanding Achievement Award from the IEEE Industry Applications Society (IAS), the 1990 William E. Newell Award of the IEEE Power Electronics Society, and the 1995 Nicola Tesla IEEE Field Award from the IEEE Power Engineering Society for his work. He has served the IEEE in various capacities for three IEEE Societies, including President of the IAS. He is a Fellow of the Institution of Electrical Engineers, U.K., and the Royal Academy of Engineering (Great Britain), and a Member of the Institute of Electrical Engineers of Japan. He has received 21 IEEE Prize Paper Awards from three different IEEE Societies including Best Paper Awards for publication in the IEEE TRANSACTIONS in the years 1984, 1994, and 1999. 